\title{
EVOLUTION OF THE MAGNETIC FIELD CONFIGURATION IN AN ACTIVE REGION
}

\author{
DENNIS L. SCHATZ* \\ CSIRO National Standards Laboratory, Division of Physics, Sydney, Australia
}

\begin{abstract}
Described and discussed is the evolution of the magnetic field configuration in an Active Region from observations made with high time resolution.
\end{abstract}

\section{Introduction}

At the Culgoora optical observatory a solar magnetograph of unique design using three fabry-perot interferometers in series has been developed (Giovanelli, 1967; Ramsay, 1967). The design of the instrument is such that an area of the Sun approximately $8 \mathrm{~min}$ in diameter can be observed through a tunable filter with a bandpass of $\frac{1}{20} \AA$. In conjunction with a polarizing beam-splitter and $\frac{1}{4}$ wave plate, simultaneous observations can be made in both circular polarized components. Using a technique similar in principle to Leighton's method of photographic subtraction (Leighton, 1959), a representation of the magnetic field can be obtained. Consequently, the Culgoora magnetograph offers a fast method of obtaining the magnetic configuration over a large area of the Sun.

\section{Observations}

In order to take advantage of the high time resolution allowed by this instrument, observations were made to obtain the configuration of the magnetic field in an Active Region at short time intervals and to see how the configuration evolved. The observations discussed in this paper were made on 9 and 10 February 1970. On both days observations were started at approximately 2300 UT (observations actually began 8 and 9 February UT) and ended at approximately 0700 UT. Exposures were taken approximately every two minutes with several large time gaps interspersed throughout the day. Figure 1 shows the magnetic field configuration of the active region studied near the time that the observations commenced on each day. On 9 February the sunspot was located at N19 E25 at 2200 UT (February 8) and on 10 February it was located at N19 E12 at 2200 UT (February 9).

All observations were made in the light of CaI $6102.8 \AA$, and the magnetic configuration was observed to be independent of the filter position in the blue wing of the line profile over a wavelength range which varied from the wavelength corresponding to a position on the line profile $\frac{1}{8}$ of the line depth from the line center to the wavelength corresponding to $\frac{5}{8}$ of the line depth from line center. The observations were made near the center of this range.

Each exposure simultaneously produces two images in opposite circular polar-

* Now at the Space Sciences Laboratory, University of California, Berkeley, California. 
ized light on $35 \mathrm{~mm}$ Kodak Kodalith film. These two images must then be photographically subtracted to give the desired magnetogram. The basic method used is the same one used by Leighton (1959). However only the first subtraction is carried out rather than the three made by Leighton. This is quite sufficient for our purpose since we are only concerned with the qualitative aspects of the field configuration.

As this observing program produced a large number of photographs to be subtracted, it was necessary to develop a means of subtracting photographs on a mass production

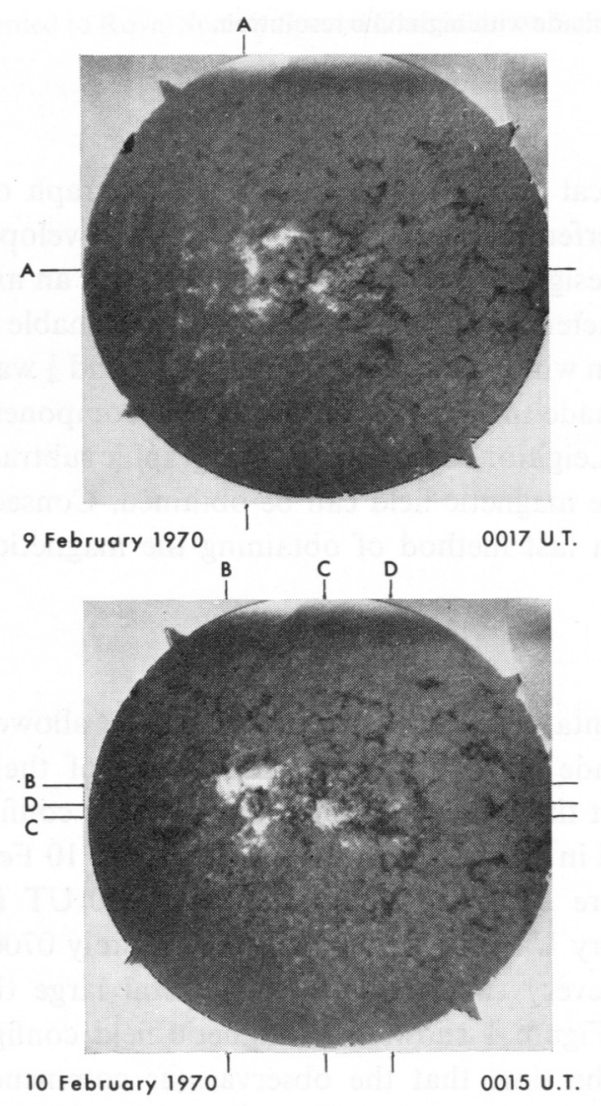

Fig. 1. Magnetic field configuration of active region near the beginning of observing period.

basis. This was done with the help of Mr. H. Gillett in the Division's photographic laboratory and a commercial film company (Supreme Films Pty. Ltd.) using their optical printer. The optical printer is a pin registered system which can accurately project the image of the negative of one of the two images in a pair on the positive of the second image. A third film is then exposed in optical contact with the second film giving the desired magnetogram. The final film is then developed to $\gamma=1.0$ with an accuracy that guarantees the $\gamma$ along the length of the entire film to vary by only \pm 0.05 .

By reregistering the magnetograms to eliminate the movement of the active region 
due to solar rotation, a cine film was produced in order to study the evolution of the magnetic field configuration. This was then projected and could be studied one frame at a time or accelerated to a rate of motion showing the evolution at 2500 times the original speed.

\section{Results}

A study of the CaI magnetograms reveals a number of structures that should be noted. There are fields which are observed to be associated with sunspots. These can be seen at points A, B, and C in Figure 1. Likewise there are fields which are not associated with sunspots, but are associated with the white light faculae (Chapman and Sheeley, 1968; Giovanelli, 1970). This association is further supported by the fact that the conglomerate of magnetic granules away from the sunspots which are observed in the magnetogram have been shown to have approximately the same size and lifetime as the white light facular granules (Schatz, 1970). We also see one sunspot whose magnetic field appears to be insensitive to the magnetograph (point D, Figure 1). However, at the same time magnetic structures, which appear to be associated with facular fields near the spot, encroach in upon the spot and appear to cover a portion of the penumbra. Further observations with the magnetograph are necessary before the nature of this overlying material can be understood.

If we now look at the motions which are observed when we examine the cine film for each day, we again encounter a number of different phenomena that should be noted. These motions can best be divided into five types:

(1) Transverse movement of field on large scale (movement $>30^{\prime \prime}$ ).

(2) Intrusion of one polarity on the opposite polarity.

(3) Disappearance of one polarity with accompanied appearance of opposite polarity.

(4) Disappearance or appearance of just one polarity.

(5) Small scale movement of individual magnetic granules or conglomerate of magnetic granules.

In Figure 2, we can easily see the transverse movement of field on a large scale (pts. A, B). This is clearly associated with expansion of sunspots underlying these points. It is interesting to note that as these spots expand they tend to clear a path through the surrounding magnetic field and push the dark polarity features to one side. This apparently causes a greater concentration of dark polarity field to be built up in between the two areas of expansion (pt. C, Figure 2). Likewise, we see the same type of large scale expansion at point A in Figure 3. Again, we see the same phenomena where the darker polarity features are swept aside. As before, this motion can be associated with the expansion of sunspots.

The intrusion of one polarity upon an area of opposite polarity is most clearly seen at pt. $\mathrm{D}$ in Figure 2. At the beginning of the day, there is a large dark polarity object bordered by two much smaller bright polarity objects. As the day progresses, the most western bright polarity object begins to intrude into the dark polarity object first, and then later the more eastern bright polarity object begins to intrude. This 


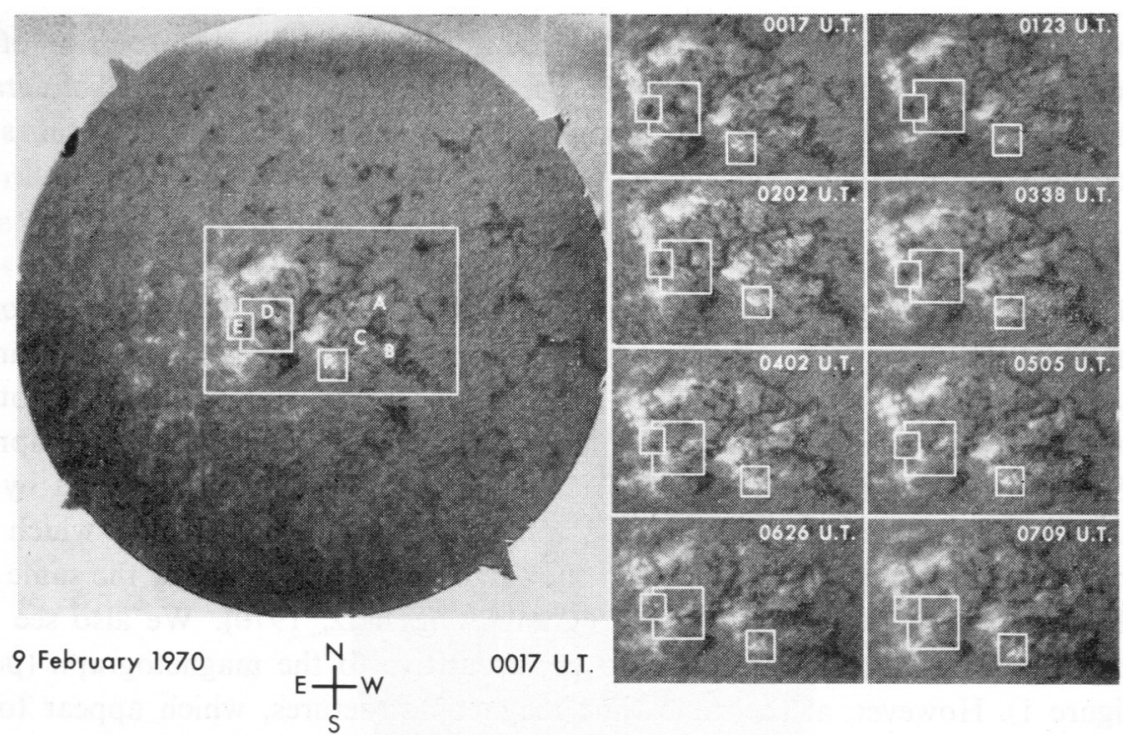

Fig. 2. Evolution of magnetic field configuration on 9 February 1970.

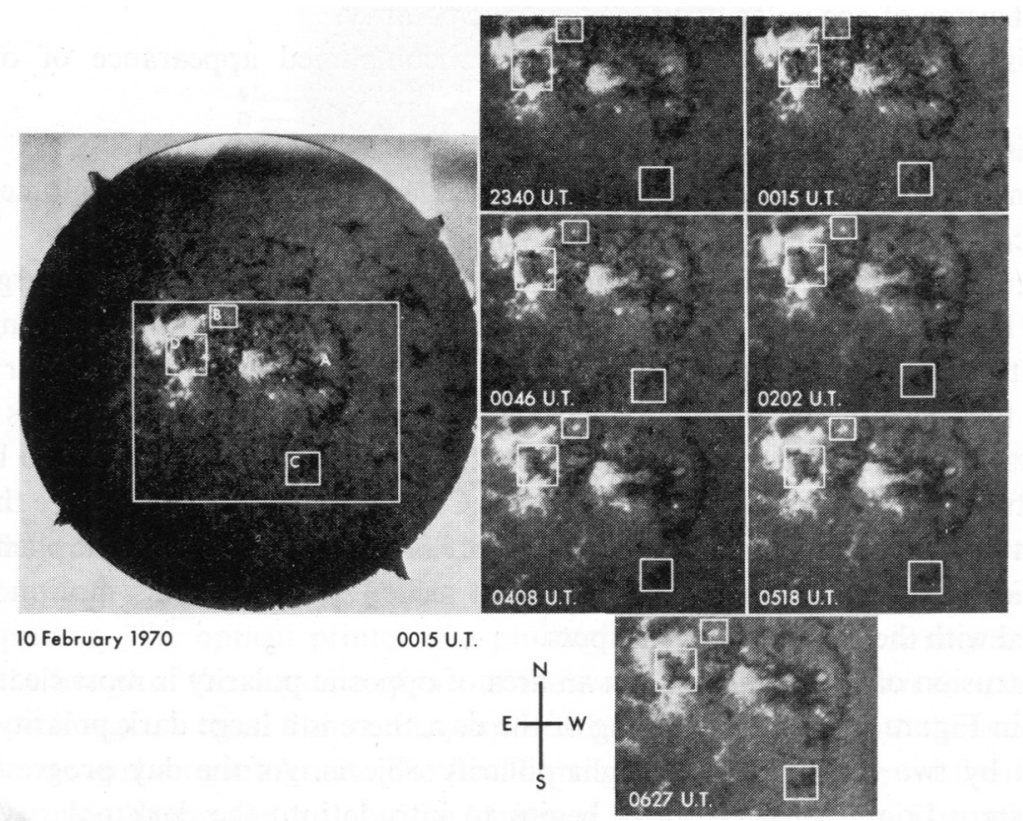

Fig. 3. Evolution of magnetic field configuration on 10 February 1970. 
continues until the end of the day when the bright fields have apparently connected and the dark field has almost completely disintegrated.

This phenomenon is very similar to the next type of evolution observed, the disappearance of one polarity with the simultaneous appearance of the opposite polarity nearby. This is seen at pt. E in Figure 2. The dark polarity object is very dark at the beginning of the observations, but slowly declines in intensity throughout the day. By 0625 UT, the dark field has almost disappeared and a bright object has developed in its place or just adjacent to it. By $0710 \mathrm{UT}$, the dark object has completely disappeared and only the bright object is left.

It is difficult to determine from the white light observations whether these changes are accompanied by development of small sunspots or whether they are associated with the facular regions. Observations of the magnetic fields near the limb with periodic observations made by tuning the magnetograph into the continuum would be necessary to resolve this question.

At the same time, there are occasions where the appearance or disappearance of fields is not accompanied by a clear cut change in the opposite polarity nearby. In Figure 2 (pt. F) we see the slow emergence of a bright polarity object as the day progresses without any change in a dark polarity which could be clearly associated with its emergence. In Figure 3 (pt. B) we see the development of a bright feature which appears during one of the gaps in our observations, but also has no clear opposite polarity evolution associated with it.

In addition to the large scale transverse movement stated earlier, there are clear examples of movement on a smaller scale. At pt. $\mathrm{C}$ in Figure 3 we can see three isolated granules that come together during the day and coalesce into two granules, and then finally into one large feature. At the same time these features as a unit have been moving closer to the larger conglomerate of magnetic field lying to the west of the three features. At pt. D, Figure 3, we see a dark polarity feature developing along a line going from $\mathrm{N}$ to $\mathrm{S}$. When this developing feature is confronted by an object of opposite polarity, it appears to spread out along the northern perimeter of the object. This development is different from the feature at pt. F (Fig. 2) since it appears to be due to the actual emergence of magnetic field rather than just transverse movement.

\section{Discussion}

Sheeley (1969) in his study of the photospheric network noted the disappearance of magnetic field was a 'mild process' at the level of the photosphere at which he was observing. This is confirmed in this study since at no time is there dramatic change in the field configuration. At the same time he speculates that if the disappearance of magnetic field was accomplished by field annihilation then we should see the simultaneous disappearance of fields of opposite polarity. He found no clear examples of this in his observations, and in agreement with this (although this is only an introductory study) we found no clear observations of the simultaneous disappearance of opposite polarity fields. 
On the contrary, there seems to be a number of occasions where there is the appearance or increase of one polarity and the disappearance or decrease of the opposite polarity adjacent to it or in place of it. (See Figure 3, pt. D and E.) It is interesting to note that this is the type of evolution observed by Ribes (1969) for EMF regions near the location of flares.

The description of the evolution seen in these high time resolution observations is a beginning to the possibilities that are open with observations of this sort. It would be very useful to compare, on a comparable time scale, the changes which occur in the chromosphere in $\mathrm{H} \alpha$ with the changes in the magnetic field configuration. The changes which occur during flare activity, surges or active filaments can now be more easily studied. In addition, the velocity of features in the chromosphere can be compared to motions of the magnetic field.

\section{References}

Chapman, G. A. and Sheeley, N. R.: 1969, Solar Phys. 9, 347.

Giovanelli, R. G.: 1967, Proc. Astron. Soc. Australia 1, 39.

Giovanelli, R. G.: 1970, Proc. Astron. Soc. Australia 1, 363.

Leighton, R. B.: 1959, Astrophys. J. 130, 366.

Ramsay, J. V.: 1967, Proc. Astron. Soc. Australia 1, 66.

Ribes, E.: 1969, Astron. Astrophys. $2,316$.

Schatz, D.: 1970, Proc. Astron. Soc. Australia 1, 371.

Sheeley, N. R.: 1968, Solar Phys. 5, 442. 